1 Fundação Oswaldo Cruz (Fiocruz), Instituto Aggeu Magalhães - Recife (PE), Brasil.

Orcid: https://orcid. org/0000-0002-24079425

meriellybezerra@hotmail. com

2 Fundação Oswaldo Cruz (Fiocruz), Instituto Aggeu Magalhães - Recife (PE), Brasil.

Orcid: https://orcid. org/0000-0002-75184137

kmedeiros@cpqam.fiocruz.br

\section{Limites do Programa de Melhoria do Acesso e da Qualidade da Atenção Básica (PMAQ-AB): em foco, a gestão do trabalho e a educação na saúde}

\author{
Limits of the National Program for Access and Quality Improvement \\ in Primary Care (PMAQ-AB): on focus, work management and \\ health education
}

Merielly Mariano Bezerra', Katia Rejane de Medeiros²

DOI: 10.1590/0103-11042018S213

RESUMO O artigo analisa os limites das subdimensões propostas pelo Programa Nacional de Melhoria do Acesso e da Qualidade da Atenção Básica (PMAQ-AB) para avaliação externa das equipes de Atenção Básica, a partir das diretrizes e dos princípios do campo Gestão do Trabalho e da Educação em Saúde (GTES). Realizou-se um estudo descritivo, com abordagem qualitativa e análise documental sobre o PMAQ-AB, elegendo-se o instrumento de avaliação externa e publicações do Ministério da Saúde relacionadas à política de GTES, no período de 2011 a 2014. Constatou-se que o instrumento de avaliação do PMAQ-AB converge com a política de GTES nos aspectos da precarização do trabalho e da valorização do trabalhador, porém, o instrumento não explora a negociação do trabalho e a saúde do trabalhador. Sua concepção de Educação Permanente em Saúde (EPS) é limitada, embora apresente potencialidades quando investiga a relação das ações de EPS ofertadas e as necessidades das equipes e ao retratar a integração ensino-serviço como um padrão de qualidade. Afirma-se que há necessidade de aprimoramento dos instrumentos do PMAQ-AB para o fortalecimento das diretrizes e dos princípios das políticas do GTES para o Sistema Único de Saúde (SUS), contudo, assume-se que o Programa tem potencial de geração de informações para o sistema de saúde.

PALAVRAS-CHAVE Atenção Primária à Saúde. Avaliação em saúde. Gestão em saúde. Recursos humanos em saúde.

ABSTRACT The article analyzes the limits of the sub-dimensions proposed by the National Program for Access and Quality Improvement in Primary Care (PMAQ-AB) for the external evaluation of the Primary Care teams, based on the guidelines and principles of the field of Work Management and Health Education (GTES). A descriptive study, with a qualitative approach and documentary analysis on the PMAQ-AB was carried out, and the external evaluation instrument and publications of the Ministry of Health related to the policy of the GTES were selected, from 2011 to 2014. It was observed that the evaluation instrument of the PMAQ-AB converges with the GTES policy in the aspects of work precarization and worker valorization, however, the instrument does not exploit work negotiation and worker health. Its conception of Permanent 
Health Education (EPS) is limited, although it presents potentialities when it investigates the relation of the EPS actions offered and the needs of the teams and when portraying the teaching-service integration as a quality standard. It is stated that there is a need to improve the $P M A Q-A B$ instruments in order to strengthen the guidelines and principles of the policies of the GTES for Unified Health System (SUS), however, it is assumed that the Program has the potential of generating information for the health system.

KEYWORDS Primary Health Care. Health evaluation. Health management. Health manpower.

\section{Introdução}

No Brasil, no campo das políticas públicas, o status de autonomia dos estados e municípios na federação exige da União capacidade de induzir políticas e de implementar medidas de monitoramento e avaliação de seus entes. Pelas especificidades e tendência de mudanças no setor saúde, tais imposições são ainda mais relevantes $\mathbf{1}$. Reconhecendo a necessidade de institucionalização da avaliação em saúde, o Ministério da Saúde (MS), com a Portaria $\mathrm{n}^{0} 1.654$, estabeleceu o Programa de Melhoria do Acesso e da Qualidade da Atenção Básica (PMAQ-AB), que, além de buscar garantir um padrão de qualidade comparável nacional, regional e localmente, dando maior transparência e efetividade às ações de governo direcionadas a esse nível de atenção, também possibilita incentivo financeiro, denominado Componente de Qualidade do Piso de Atenção Básica (PAB).

Com quatro fases distintas, que se complementam e conformam num ciclo contínuo de melhoria do acesso e da qualidade, o PMAQ-AB é iniciado com: 1) processo de adesão e contratualização de indicadores entre as equipes de saúde e os gestores; 2 ) fase de desenvolvimento, que envolve as ações a serem realizadas para promover movimentos de mudança da gestão, do cuidado e da gestão do cuidado; 3) avaliação externa, quando realiza-se um conjunto de ações para averiguar as condições de acesso e de qualidade dos participantes; e, por último, 4) o processo de recontratualização das equipes e dos gestores, com o incremento de novos padrões e indicadores de qualidade, estimulando a institucionalização de um processo cíclico e sistemático, a partir dos resultados alcançados pelos participantes do Programa2-4 .

É pertinente ponderar os aspectos macropolíticos do PMAQ-AB, quais sejam: um modelo de avaliação de desempenho que propõe mensurar efeitos da política de saúde, visando a vincular o resultado da avaliação de desempenho das equipes a repasses de recursos financeiros para o gestor municipal e, consequentemente, mobilizar a tomada de decisão para promoção da melhoria da qualidade e do acesso(3,5,6).

Deve-se considerar a forma de avaliação de desempenho nele proposta, que contempla os trabalhadores entrevistados pelo programa, que são representantes de suas respectivas equipes.

Sobre os aspectos que envolvem a abordagem metodológica do PMAQ-AB, há várias opiniões. Fausto et al. ${ }^{6}$ apontam que o Programa se aproxima de um tipo de avaliação colaborativa e construtivista, onde partes interessadas no processo, ainda que não compartilhem das mesmas intenções e projetos, possibilitam reconhecer e incorporar a avaliação de questões e preocupações de consenso, conferindo maior pertinência e 
tornando mais real a permeabilidade do processo frente às responsabilidades assumidas pelos atores interessados.

Contudo, o caráter competitivo do Programa está explícito em seu modo de configuração do pagamento do componente de qualidade do PAB variável, fundo a fundo, em função do desempenho obtido pelas equipes da Atenção Básica (AB), embora, a priori, o $\mathrm{PMAQ}-\mathrm{AB}$ não preveja um sistema de recompensas que alcance diretamente o trabalhador da equipe de AB. Pode-se dizer que a forma de concorrência estabelecida no Programa é entre equipes. Logo, denota-se a possibilidade de pressão e cobrança exercida pelos pares, entre os trabalhadores, uma vez que o desempenho individual de um trabalhador pode comprometer o resultado final de toda a equipe ${ }^{7}$.

Como uma estratégia nacional de avaliação da Política Nacional de Atenção Básica (PNAB), o PMAQ-AB está organizado através de dimensões estratégicas de um conjunto de padrões de qualidade esperados para: estrutura, processos e resultados ${ }^{8}$, refletindo, portanto, características relacionadas aos programas e às políticas do Sistema Único de Saúde (SUS). Logo, afirma-se que o Programa deve se pautar nos princípios e diretrizes das várias políticas do SUS.

Nessa perspectiva, Pinto, Sousa e Ferla ${ }^{9}$ consideram que o PMAQ-AB é, entre as diversas medidas que conformam a PNAB, aquela que a sintetiza melhor, pois se articula com diversas iniciativas, estabelecendo com elas uma relação de síntese e sinergia.

Sabe-se que as formas e as condições de trabalho, bem como os processos de formação e qualificação profissional são dimensões que interferem diariamente no modo como os trabalhadores desenvolvem a produção do cuidado em saúde, e, por isso, devem ser consideradas transversais e norteadoras da AB. A exploração e a disponibilidade de dados contidos nos instrumentos do PMAQ-AB colocam-se, então, como oportunas frente à necessidade de avaliar tais dimensões na $\mathrm{AB}$.

Reconhecendo a potencialidade de análise do campo do trabalho e da educação na saúde através do PMAQ-AB, Seidl et al. ${ }^{10}$ indicam que existe uma aproximação do cenário da gestão do trabalho nas equipes participantes do PMAQ-AB no que se refere aos aspectos de qualificação, educação permanente, contratação, vínculo e carreira. Com argumentos semelhantes, Rizzotto et al."1 analisaram os dados do PMAQ-AB sobre a gestão do trabalho e da educação na saúde no estado do Paraná e evidenciam a necessidade de priorização de ações voltadas ao fortalecimento de políticas mais efetivas nesse campo no âmbito da gestão da AB do SUS.

Contudo, sobressai a necessidade de refletir sobre a estrutura propositiva do instrumento PMAQ-AB e de identificar seus limites críticos. Apoiando-se nessa premissa, neste artigo, analisam-se as subdimensões propostas pelo PMAQ-AB frente às diretrizes e aos princípios da política de Gestão do Trabalho e da Educação em Saúde (GTES).

\section{Material e métodos}

Fez-se um estudo descritivo, com abordagem qualitativa, adotando-se análise documental12. Analisaram-se documentos que normatizam e regulamentam o PMAQ-AB, que se encontram em domínio público irrestrito, selecionados no site http://dab.saude. gov.br/portaldab/ape_pmaq.php; e publicações do MS relacionadas à política de GTES. Selecionaram-se documentos de 2011 a 2014, justificados pelo fato de ser de 2011 a instituição do PMAQ-AB, e 2014 o ano de realização da avaliação externa do segundo ciclo, com a respectiva disponibilização pública dos dados, os quais foram o instrumento de análise. Foram examinados 04 documentos (02 manuais e 02 instrumentos de avaliação externa) do PMAQ-AB e 10 portarias, descritos no quadro 1 . 
Quadro 1. Distribuição dos documentos relacionados ao PMAQ-AB analisados

\begin{tabular}{|c|c|}
\hline ANO & PUBLICAC̣ÃO \\
\hline 2011 & $\begin{array}{l}\text { Portaria № 1.654, de } 19 \text { de julho - Institui, no âmbito do SUS, o PMAQ-AB e o seu Incentivo Financeiro, } \\
\text { denominado Componente de Qualidade do Piso de Atenção Básica Variável - PAB Variável. }\end{array}$ \\
\hline 2011 & $\begin{array}{l}\text { Portaria № 576, de } 19 \text { de setembro - Estabelece novas regras para a Carga Horária Semanal (CHS) dos } \\
\text { profissionais médicos, enfermeiros e cirurgião-dentista. }\end{array}$ \\
\hline 2011 & $\begin{array}{l}\text { Portaria № 2.812, de } 29 \text { de novembro - Homologa a adesão dos Municípios e das respectivas equipes de } \\
\text { Atenção Básica ao PMAQ-AB. }\end{array}$ \\
\hline 2011 & $\begin{array}{l}\text { Portaria № 225, de } 10 \text { de fevereiro - Homologa a adesão dos Municípios e das respectivas equipes de } \\
\text { Atenção Básica ao PMAQ-AB. }\end{array}$ \\
\hline 2011 & $\begin{array}{l}\text { Portaria № } 644 \text {, de } 10 \text { de abril - Homologa a adesão dos Municípios e das respectivas Equipes de Aten- } \\
\text { ção Básica ao PMAQ-AB. }\end{array}$ \\
\hline 2011 & $\begin{array}{l}\text { Portaria № 1.089, de } 28 \text { de maio - Define o valor mensal integral do incentivo financeiro do PMAQ-AB, } \\
\text { denominado como Componente de Qualidade do Piso de Atenção Básica Variável (PAB Variável). }\end{array}$ \\
\hline 2012 & $\begin{array}{l}\text { Documento - Manual Instrutivo - SAÚDE MAIS PERTO DE VOCÊ - ACESSO E QUALIDADE - PROGRA- } \\
\text { MA NACIONAL DE MELHORIA DO ACESSO E DA QUALIDADE DA ATENÇÃO BÁSICA (PMAQ). }\end{array}$ \\
\hline 2012 & $\begin{array}{l}\text { Documento - Instrumento de Avaliação Externa - ACESSO E QUALIDADE - PROGRAMA NACIONAL DE } \\
\text { MELHORIA DO ACESSO E DA QUALIDADE DA ATENÇÃO BÁSICA (PMAQ). }\end{array}$ \\
\hline 2013 & $\begin{array}{l}\text { Portaria № 261, de } 21 \text { de fevereiro - Institui, no âmbito da Política Nacional de Saúde Bucal, o Programa de } \\
\text { Melhoria do Acesso e Qualidade dos Centros de Especialidades Odontológicas (PMAQ-CEO) e o Incenti- } \\
\text { vo Financeiro (PMAQ-CEO), denominado Componente de Qualidade da Atenção Especializada em Saúde } \\
\text { Bucal. }\end{array}$ \\
\hline 2013 & $\begin{array}{l}\text { Portaria № } 283 \text {, de } 28 \text { de fevereiro - Autoriza o repasse do incentivo financeiro do PMAQ-AB, denomina- } \\
\text { do como Componente de Qualidade do Piso de Atenção Básica Variável. }\end{array}$ \\
\hline 2013 & Portaria № 635, de 17 de abril - Homologa a adesão dos Municípios ao segundo ciclo do PMAQ. \\
\hline 2013 & $\begin{array}{l}\text { Documento - Instrumento de Avaliação Externa - Instrumento de Avaliação Externa para as Equipes de } \\
\text { Atenção Básica (Saúde da Família e Equipe Parametrizada). }\end{array}$ \\
\hline 2013 & $\begin{array}{l}\text { Documento - Manual Instrutivo - SAÚDE MAIS PERTO DE VOCÊ - ACESSO E QUALIDADE - PROGRA- } \\
\text { MA NACIONAL DE MELHORIA DO ACESSO E DA QUALIDADE DA ATENCCÃO BÁSICA (PMAQ) - (Saú- } \\
\text { de da Família, Saúde Bucal e Equipes Parametrizadas) e NASF. }\end{array}$ \\
\hline 2014 & $\begin{array}{l}\text { Portaria № 2.666, de } 4 \text { de dezembro - Autoriza o repasse do incentivo financeiro do PMAQ-AB, denomi- } \\
\text { nado como Componente de Qualidade do Piso de Atenção Básica Variável. }\end{array}$ \\
\hline
\end{tabular}

Fonte: Elaborada própria.

$\mathrm{Na}$ análise documental, buscou-se identificar os seguintes aspectos: as dimensões representativas da Gestão do Trabalho e da Educação na saúde, propostas na avaliação externa das equipes da Estratégia Saúde da Família (ESF) pelo PMAQ-AB no seu segundo ciclo, para posterior composição de categorias de análise.

$O$ processo de avaliação externa do PMAQ-AB (técnica de entrevista), no segundo ciclo do Programa, teve o instrumento de coleta, dividido em três módulos: Módulo I - Observação na Unidade Básica de Saúde (UBS), cujas questões foram direcionadas para a realização do censo de infraestrutura da unidade de saúde; Módulo II - Entrevista com profissional de saúde de nível superior sobre processo de trabalho da equipe de $A B$ e verificação de documentos da UBS; Módulo III - Entrevista com o usuário na UBS sobre satisfação e condições de acesso e utilização de serviços de saúde ${ }^{4}$. Esse instrumento é composto por 893 padrões de qualidade, que refletem necessidades de informações acerca da PNAB para subsidiar a formulação e/ou os aprimoramentos das políticas e dos programas relacionados, além da certificação das equipes participantes do PMAQ-AB ${ }^{4,8}$. Sendo, portanto, o documento eleito para a análise realizada neste estudo. 
No que concerne a princípios e diretrizes da GTES, no instrumento (Módulo II), identificaram-se 55 padrões de qualidade relacionados. São as subdimensões: informações sobre o entrevistado, formação e qualificação dos profissionais da equipe de atenção básica, vínculo, plano de carreira e educação permanente do processo de qualificação das ações desenvolvidas (quadro 2).

Quadro 2. Distribuição do número de variáveis selecionadas para estudo, segundo as subdimensões do Módulo II, estabelecidas pelo instrumento de coleta da avaliação externa no segundo ciclo do PMAQ-AB.

\begin{tabular}{ll}
\hline SUBDIMENSÃO & $\begin{array}{l}\text { NÚMERO DE VARIÁVEIS } \\
\text { (PADRÕES DE QUALIDADE) }\end{array}$ \\
\hline 3 - Informações sobre o entrevistado & 02 \\
4 - Formação e qualificação dos profissionais da equipe de atenção básica & 32 \\
5 - Vínculo & 03 \\
6 - Plano de carreira & 05 \\
7 - Educação permanente do processo de qualificação das ações desenvolvidas & 12 \\
\hline
\end{tabular}

Fonte: Elaborada própria.

A composição de categorias de análise considerou as dimensões representativas da GTES: Norma Operacional Básica de Recursos Humanos para o SUS (NOB/RHSUS), princípios e diretrizes, as diretrizes operacionais do Pacto de Gestão/Pacto pela Saúde, as diretrizes e os itens da Agenda da Secretaria de Gestão do Trabalho e da Educação na Saúde (SGTES) e a Política Nacional de Educação Permanente em Saúde (PNEPS), todas selecionadas para análise, por serem basilares para a política Assim, chegou-se às seguintes categorias: Precarização do trabalho; Valorização e Negociação; Educação Permanente; Relação da $\mathrm{AB}$ com Instituições de Ensino.

Salienta-se que o artigo integra parte dos resultados da pesquisa multicêntrica 'A Política de Educação Permanente para trabalhadores da ESF em Pernambuco, Rio de Janeiro e Bahia: a análise dos trabalhadores e gestores', apoiada pelo edital PPSUS - Pernambuco (CNPq/MS/SES/Facepe 10/2017) e aprovada pelo Comitê de Ética em Pesquisa do Centro de Pesquisas Aggeu Magalhães, sob o n ${ }^{0}$ 70465917.2.0000.5190.

\section{As subdimensões do instrumento PMAQ-AB e as diretrizes e os princípios da política de Gestão do Trabalho e da Educação em Saúde: limites críticos}

Já destacada a centralidade GTES como eixo estruturante do SUS e considerando que essas questões relacionadas às equipes de $\mathrm{AB}$ carecem de maior aprofundamento, buscou-se discuti-las a partir das categorias de análise propostas.

\section{Precarização do trabalho}

O instrumento de avaliação externa do PMAQ-AB permite uma análise de dados relacionados à precarização do trabalho na AB. A partir de informações relacionadas ao vínculo (subdimensão II), pode-se obter: agente contratante, tipo de vínculo e forma de ingresso no serviço público, que possibilita apontar certo cenário de terceirização e flexibilização da força de trabalho nessa área. 
A questão relativa à precarização do trabalho se constitui como preocupação e objeto de pesquisas de diversos campos do conhecimento, bem como tema presente na agenda de diversos atores sociais e órgãos governamentais nacionais e internacionais ${ }^{13-16}$. Na saúde, o tema tem sido apontado como um desafio no SUS 10,11,13,14. Neste sentido, ressalta-se como relevante a oportunidade de análise do problema a partir do PMAQ-AB.

Consoante às reflexões, é importante a compreensão da abrangência da noção de trabalho precário no SUS, que se caracteriza pela ausência de proteção social do trabalho, havendo perdas de direitos que são assegurados constitucionalmente, onde outras questões podem se encontrar nessa conceituação, tais como baixos salários, condições inadequadas de trabalho e excesso de jornada de trabalho ${ }^{17}$. Tais fatores caracterizam a fragilidade dos direitos trabalhistas, são condicionantes de estresse e, consequentemente, influenciam a qualidade dos serviços prestados aos usuários ${ }^{18}$.

Para o MS, semelhante à compreensão das entidades sindicais, o trabalho precário é aquele realizado sem a proteção social do trabalhador e engloba todo trabalho realizado sem concurso público. O Conselho Nacional dos Secretários de Saúde (Conass) e o Conselho Nacional dos Secretários Municipais de Saúde (Conasems) defendem a flexibilização do trabalho em algumas situações. Para esses coletivos, o trabalho precário relaciona-se aos vínculos de trabalho no SUS que não garantem os direitos trabalhistas e previdenciários consagrados em lei, seja por meio de vínculo direto ou indireto ${ }^{19}$.

Voltando para o instrumento do PMAQ$\mathrm{AB}$, há identificação do agente contratante do profissional. Isso possibilita a verificação da existência de diferentes formas de gestão na contratação dos profissionais da $\mathrm{AB}$, através de dados: administração direta, consórcio intermunicipal (de direito público e/ou de direito privado), fundação pública (de direito público e/ou de direito privado), Organizações sociais (OS), Organização da Sociedade Civil de Interesse Público (Oscip), entidade filantrópica, Organização não Governamental (ONG), empresa, cooperativa ou outros.

Soma-se a isso o reconhecimento da forma de ingresso e do tipo de vínculo dos profissionais, indicativas de prerrogativas ao trabalho no SUS, que se apresentam como potencialidades de análise do Programa para área da gestão do trabalho.

Tais aspectos apontam para sinais da flexibilização do trabalho, mas não representam necessariamente a caracterização da situação de alcance da proteção aos direitos trabalhistas e previdenciários na $\mathrm{AB}$, tendo em vista que a contratação pela administração direta não é suficiente para analisar a maior ou menor flexibilização das relações de trabalho, posto que não garante as formas mais protegidas de vínculo ${ }^{10}$.

De modo geral, o tema da precarização do trabalho costuma ser discutido em conjunto com a flexibilização das leis trabalhistas. No caso do SUS, incluindo a $\mathrm{AB}$, a precarização dos vínculos e da regulação das rela-ções de trabalho tem algumas particularidades, tendo em vista a desestruturação do aparato de Estado e de suas carreiras ocorrida nas últimas décadas e dos constrangimentos impostos pelo contexto de restrição fiscal ${ }^{15}$.

A flexibilidade, traduzida pelo redesenho da relação entre o público e o privado, na área da saúde, tem assumido conformações várias: modificações na estrutura jurídico-administrativa de unidades prestadoras de serviço de saúde e ter-ceirizações de atividades relativas à gestão 1 .

O Programa Nacional de Desprecarização do Trabalho no SUS (DesprecarizaSUS), proposto pelo MS, reflete uma estratégia de enfrentamento objetivando implantar uma política de valorização do trabalhador. Foi uma parceria dos gestores estaduais, municipais e representantes de entidades sindicais ${ }^{15}$.

Considerando-se o DesprecarizaSUS e sua descentralização de ações nas esferas, cabe indagar se há uma real interferência do MS 
nos casos em que o resultado do PMAQ-AB aponte para descumprimento das formas adequadas de contratação dos trabalhadores das equipes e se são adotadas medidas, pois sabe-se que o problema repercute diretamente na proteção social ao trabalhador.

\section{Valorização e negociação do trabalho}

O PMAQ-AB favorece uma análise da valorização do trabalhador quando aborda o Plano de Cargos, Carreiras e Salários (PCCS). Nas diretrizes fixadas na NOB/RH-SUS, o PCCS é considerado um instrumento de ordenação do trabalho, devendo ser incorporado em cada nível de gestão do SUS ${ }^{15}$.

Como instrumento de gestão do trabalho, o PCCS propõe valorizar o trabalhador através de um conjunto de normas que orienta e disciplina a trajetória do trabalhador em sua carreira, com a respectiva remuneração, favorecendo a qualificação profissional15,20.

Nessa dimensão, para além da disposição de PCCS, o PMAQ-AB questiona os critérios de progressão: por antiguidade, avaliação de desempenho e/ou desenvolvimento (mérito) e por titulação/formação profissional. Essas questões possuem pertinência e dialogam com as proposições das diretrizes nacionais para a instituição de PCCS-SUS.

Apesar de a discussão do PCCS-SUS não ser recente, é atual frente à necessidade de consolidar essa estratégia, sendo discutida na maioria dos municípios no âmbito do SUS. Essa realidade é motivada pela existência de trabalhadores de saúde que, em sua grande maioria, desenvolvem atividades em nível precário, com irregularidades trabalhistas que vão desde a contratação temporária até a falta de uma política salarial definida ${ }^{\mathbf{1 0}}$.

Em 2004, o MS iniciou a discussão sobre a necessidade de elaboração de diretrizes para subsidiar a elaboração dos PCCS dos trabalhadores do SUS nas três esferas, com o pressuposto de construir carreiras semelhantes no âmbito dos sistemas, respeitando-se as especificidades regionais ${ }^{\mathbf{1 1}}$.
Em 2006, a Mesa Nacional de Negociação Permanente do SUS (MNNP-SUS) estabeleceu as Diretrizes Nacionais para a instituição de planos (DNPCCS-SUS) e definiu princípios, servindo de referência para a análise dos PCCS: universalidade dos planos; equivalência dos cargos ou empregos; concurso público como única forma de ingresso; mobilidade; flexibilidade; gestão partilhada de carreira como garantia de participação dos trabalhadores; carreira como instrumento de gestão; educação permanente; avaliação do desempenho; e compromisso solidário, em prol da qualidade dos serviços, do profissionalismo e da adequação técnica do profissional às necessidades do serviço de saúde ${ }^{\mathbf{1 8}}$.

Apesar de propor ações de sensibilização entre os gestores, com vista à elaboração e à implantação de uma nova política de recursos humanos, a implantação DNPCCS-SUS, em muitos municípios, esbarrou em questões como impacto financeiro e restrições impostas pela Lei de Responsabilidade Fiscal ${ }^{10}$.

É possível identificar, ainda, como estímulo à implantação do PCCS pelo MS, o lançamento do tema Carreira: Planos de Cargos, Carreiras e Salários no edital para o projeto InovaSUS-Carreira, em 2012. Na oportunidade, foram premiadas 12 iniciativas. $\mathrm{O}$ objetivo era de valorizar as propostas de planos estabelecidas pelos sistemas locais, mas também impulsionar novas proposituras ${ }^{\mathbf{1 9}, \mathbf{2 1}}$.

Outro ponto a ser destacado, no PMAQ$\mathrm{AB}$, são as formas de incentivo, gratificação ou prêmio financeiro por desempenho. Todavia, esses pontos apresentam apenas um panorama geral, sem possibilitar a análise das características e dos critérios que embasaram os possíveis programas/ações de incentivos financeiros que possam estar sendo implantados e desenvolvidos na $\mathrm{AB}$.

Pode-se indicar que o PMAQ-AB tem a capacidade de revelar pontos sobre a valorização dos profissionais, a partir da incorporação do PCCS e de outras formas de incentivo, gratificação ou prêmio financeiro por desempenho. Mas o Programa não 
dispôs da mesma potencialidade quando se trata da adoção de formas de negociação do trabalho no SUS. Na análise dos documentos relacionados ao Programa, nota-se total ausência do olhar para questões relacionadas à negociação do trabalho.

Entendendo a complexidade e a dinamicidade das relações de trabalho, particularmente delineadas na saúde, e a necessidade de buscarem-se alternativas compartilhadas entre gestores, trabalhadores e controle social, cabe ressaltar aqui a importância da negociação do trabalho como uma das pautas prioritárias da Gestão do Trabalho em Saúde (GTS).

A negociação do trabalho em saúde pode ser compreendida como diálogo político, e, como tal, é parte integrante dos processos de tomada de decisão, contribuindo para o desenvolvimento ou a implementação de mudanças de políticas de gestão do trabalho no SUS'22. Representa, também, uma forma direta, flexível e rápida de resolver conflitos coletivos ${ }^{23}$.

As ações da gestão local nos serviços do SUS têm reflexos diretos sobre as relações de trabalho porque abrem ou emperram as ações do trabalhador de saúde como sujeito e protagonista no desenrolar de suas atividades. Dito de outro modo, o processo de sindicalização, os patamares de organização dos trabalhadores e, sobretudo, a criação de espaços de negociação para o debate de suas reivindicações dependem também das margens para ação ampliadas ou restritas pelos modelos de gestão21.

Nesse movimento, a negociação coletiva no SUS se apresenta com instrumento de gestão impulsionado pelo Programa de Qualificação e Estruturação da Gestão do Trabalho e da Educação no SUS (ProgeSUS), processo alçado como ponto de pauta da agenda da SGTES após a reativação da MNNP, que objetivou garantir um espaço democrático, institucional e paritário para a negociação no âmbito das questões do trabalho no SUS22.

O MS afirma, em diversos documentos oficiais, a mesa de negociação como ferramenta de gestão, capaz de intervir de maneira positiva na efetividade dos serviços prestados aos usuários, pois busca a mediação dos conflitos e a resolução dos problemas do cotidiano do trabalho no SUS. Para tanto, estrutura-se como um fórum, nos três âmbitos, baseado nos princípios constitucionais da legalidade, publicidade e liberdade sindical, reunindo gestores, prestadores de serviços e entidades sindicais representativas dos trabalhadores da saúde. $\mathrm{O}$ processo de negociação permanente é essencial para o sucesso da política de GTS, estando pautado na NOB-RH15,19,21.

Por não abordar a negociação em seus documentos, o PMAQ-AB apresenta limites, parecendo não considerar a inserção do trabalhador em sindicatos ou outros espaços de negociação coletiva, talvez por verificar apenas o desempenho do profissional na sua atuação e não 'considerar/valorizar' a participação ou o conhecimento deste sobre as práticas de negociação coletiva disponíveis. Nesse sentido, a proposta do Programa pode fortalecer a 'desagregação' do trabalho, ou melhor, a visão de recurso e não de agente transformador quando não pontua esse aspecto como um dos integrantes da qualidade na $\mathrm{AB}$ e não estimula a cultura de negociação do trabalho.

Do ponto de vista da regulação, a diferenciação dos vínculos e a intermediação dos contratos colocam o trabalhador numa posição vulnerável na medida em que as negociações são feitas individualmente, pouco regulamentadas, e os contratos são negociados pelas empresas, cabendo ao trabalhador apenas aderir ao que já foi previamente acordado ${ }^{24}$.

Como o instrumento do PMAQ-AB reconhece a multiplicidade de agentes contratantes na $\mathrm{AB}$, com entes privados participando de forma considerável na gestão da saúde e não discutindo negociação do trabalho, afirma-se que o programa deixa uma relevante lacuna.

Emerge, também, como importante ponto de análise a saúde do trabalhador do SUS, pois sabe-se que alguns dos temas já mencionados são considerados fatores 
associados ao desenvolvimento ou à degradação da satisfação e do bem-estar dos trabalhadores de saúde.

No escopo do PMAQ-AB, a questão não aparece, configurando uma divergência com a área de GTS, visto que a saúde do trabalhador do SUS é reconhecida como aspecto da valorização do trabalhador.

A saúde do trabalhador pode ser compreendida a partir das relações, condições de trabalho e de vida dos trabalhadores, gerando processo de saúde-doença. A Política Nacional de Saúde do Trabalhador e da Trabalhadora (PNST) define os princípios, as diretrizes e as estratégias a serem observados pelas três esferas de gestão do SUS, para o desenvolvimento da atenção integral à saúde do trabalhador, com ênfase na vigilância, visando à promoção e à proteção da saúde dos trabalhadores e à redução da morbimortalidade decorrente dos modelos de desenvolvimento e dos processos produtivos $^{21}$. Mas as ações no campo da saúde do trabalhador ainda permanecem distantes das possibilidades que nela se apresentam para diagnóstico, vigilância, atenção, direito à informação, entre outros ${ }^{25}$.

O cenário de expressão da saúde e do trabalho vem sofrendo transformações, e as determinações que incidem sobre a saúde do trabalhador na contemporaneidade estão relacionadas às novas modalidades de trabalho e aos processos mais dinâmicos de produção implementados pelas inovações tecnológicas e pelas atuais formas de organização e gestão do trabalho' ${ }^{26}$.

Os aspectos relacionados à saúde do trabalhador são reconhecidos como competência do SUS e, ao mesmo tempo, como problema que afeta diretamente sua consolidação.

Em 2006, pelos menos 600 mil trabalhadores do SUS se encontravam em condições precárias de trabalho ${ }^{15}$. Em 2010, a Previdência Social registrou cerca de 720 mil acidentes, houve mais de 15 mil afastamentos do trabalho por incapacidade permanente e mais de 2.500 mortes, gerando impacto orçamentário, tendo sido gastos, em 2010 , cerca de $R \$ 11$ bilhões para pagamento de auxílio-doença e auxílio-acidente ${ }^{27}$. Os números expressam a fragilidade do instrumento PMAQ-AB quando exclui essa dimensão de análise nas equipes.

\section{Educação Permanente em Saúde (EPS)}

A formação e a qualificação do trabalhador de saúde é central, mas é também nó crítico à consolidação do SUS, em especial, na $\mathrm{AB}^{111,28,29}$.

Reconhecer as concepções e os processos de educação voltados ao trabalhador do SUS aponta necessidades de reconhecer que, ao longo do seu percurso de construção, foram vivenciadas diversas proposições teóricas e movimentos para institucionalizações de práticas da educação na saúde. Entretanto, atualmente, a que está pautada como referencial teórico e 'político' para o SUS é a proposta da EPS.

A Gestão da Educação na Saúde (GES), fundamentada nos princípios da EPS, une os processos de formação, da atenção à saúde, da gestão dos serviços de saúde e da participação ou do controle social. Essa fundamentação ampliou o número de atores com os quais os responsáveis pela GES passam a se relacionar, exigindo mecanismos de gestão participativa e o entendimento do que significa um embasamento na EPS.

A EPS deve ser compreendida, quanto aos seus pressupostos, como uma estratégia transformadora das práticas de saúde, colaborando para romper com o paradigma tradicional que orienta os processos de formação dos trabalhadores da saúde. Aponta para o desenvolvimento pessoal, social e cultural e está centrada nos processos de ensino-aprendizagem, em que o próprio sujeito que aprende é um agente ativo, autônomo e gestor de sua educação $0^{30}$.

Em 2004, institui-se a PNEPS como estratégia de formação e desenvolvimento de 
trabalhadores de saúde ${ }^{31}$. Além de definir a concepção da EPS, a PNEPS foi um avanço quando elenca diretrizes de implementação por meio da condução regional da Política e da participação interinstitucional, além de estabelecer orçamento para projetos e ações ${ }^{28}$.

É preciso reconhecer que, desde 2004, ano de publicação da PNEPS, até o momento, houve avanços e dificuldades, constituídas nos diferentes momentos políticos do País, como processos de rupturas, mudanças de enfoque e questões de (sub)financiamento, que podem ser verificados no seu processo de implementação.

Essas questões refletem no desenvolvimento da PES. Destacam-se, nesse processo: pouca articulação entre os atores (gestores, trabalhadores, Instituições de Ensino e Pesquisa IES e controle social), incipiente participação dos gestores municipais, indefinição de parâmetros para construção de projetos, ausência de avaliação dos projetos desenvolvidos no que tange às suas desejadas mudanças nas práticas de formação, gestão e atenção na saúde, dificuldades na utilização dos recursos financeiros, entre outras ${ }^{28,30}$.

Nesse ambiente se afirma a potência do PMAQ-AB em apontar dados, de forma capilarizada, das ações e EPS das equipes da $A B$, pela pertinência de avaliação das ações ou dos processos de educação, no locus de atuação dos atores ou sujeitos que operam a EPS em seu cotidiano do trabalho. Mas isso não suprime a necessidade de uma análise mais apurada das diretrizes e dos princípios, bem como possíveis ações de implementação, propostas para avaliação da EPS pelo Programa.

Amparando-se nesse argumento, a exploração dos documentos que normatizam o PMAQ-AB indica duas abordagens principais sobre EPS. A primeira aponta como integrante das dimensões da segunda fase do PMAQ-AB, chamada de fase de desenvolvimento, que envolve o conjunto de ações a serem realizadas pelas equipes e pelas gestões, no intuito de promover movimentos de mudança da gestão, do cuidado e da gestão do cuidado, que produzirão a melhoria do acesso e da qualidade da $A B^{\mathbf{2}, 4}$.

A segunda abordagem do PMAQ-AB sobre a EPS, objeto deste artigo, levanta as informações sobre EPS, através da terceira fase do PMAQ-AB, que consiste na avaliação externa das equipes participantes do programa.

O PMAQ-AB, ao mesmo tempo que busca investigar questões relativas ao tema da EPS, também procura integrar iniciativas institucionais para apoiar os processos formativos intraequipe, considerados como dispositivos para a qualificação da atenção e a implementação de mudanças apontadas pelo programa ${ }^{9}$.

A análise do instrumento de avaliação externa indica que o PMAQ-AB investigou a EPS na $A B$, de modo geral, quando interroga sobre:

1) Participação, ações realizadas e quais tipos;

2) Utilização das possíveis ofertas de apoio do Telessaúde: da segunda opinião formativa, do telediagnóstico, da tele-educação ou da teleconsultoria;

3) Se as ações de EPS contemplavam as necessidades da equipe;

4) Acolhimento de estudantes, professores e/ou pesquisadores em atividades de ensino, pesquisa e/ou extensão, a frequência destes e se as atividades desenvolvidas estão articuladas com o processo de trabalho.

Questões interrogam sobre a apropriação do conceito de EPS (1 e 3), pois baseia-se justamente no entendimento do protagonismo dos trabalhadores no processo de transformação do seu trabalho e na construção de soluções para os problemas a partir das realidades locais dos serviços de saúde.

Como alternativa, ou forma de aproximação dessa concepção, os questionamentos levantados no instrumento do PMAQ-AB 
poderiam interrogar se a equipe desenvolve ações de EPS no seu trabalho e/ou se esses atores demandam para gestão as necessidades. Essas interrogações contribuíram para minimizar a estreita concepção de EPS que o instrumento denota.

Sem negar a necessidade de afinar o entendimento sobre a EPS, destaca-se que os questionamentos propostos pelo PMAQ-AB na avaliação das equipes sugerem que há associação entre EPS e AB. Essa constatação aponta para a importância da continuidade e do incremento de ações no escopo de uma PNEPS 9 .

No que tange ao item 2, pode-se inferir que houve um 'investimento' considerável do PMAQ-AB em mensurar as ofertas e a utilização de recursos que vêm sendo disponibilizados pelo $\mathrm{MS}$ às equipes de $\mathrm{AB}$, como é o caso do Telessaúde.

A incorporação das ofertas de apoio à formação pela PNAB colabora com o trabalho local e tem um resultado que pode ser demonstrado pelos dados coletados no PMAQ-AB'.

O MS aponta a Comunidade de práticas e o Telessáude como estratégias de EPS induzidas pela PNAB e reafirmadas pelo PMAQAB. A PNAB organizou ofertas, desde a gestão, que objetivam: a aprendizagem, a busca e o uso de informação, a construção cooperativa de saberes, a constituição de comunidades de aprendizagem e rodas de debate ou estudo-ação. O desenvolvimento de tecnologias da informação e comunicação e sua introdução nos ambientes de educação e de trabalho têm crescido nos últimos anos, e a tendência é manterem-se em ascensão. Como EPS, os recursos tecnológicos disponíveis possibilitam a aproximação de profissionais interessados na resolubilidade dos serviços de saúde ${ }^{4}$.

É preciso reconhecer a potencialidade do uso de recursos tecnológicos e outras estratégias que promovam o apoio e o diálogo entre os diferentes atores do SUS, que possam resultar na incorporação de saberes e práticas. Todavia, o Programa poderia realizar essa análise a partir da identificação da existência de espaços, núcleos ou redes locais, municipais e estaduais que realizem esse apoio, a partir de uma perspectiva mais contextualizada com a realidade dos profissionais e das equipes.

Apesar do questionamento realizado pelo PMAQ-AB, descrito no item 3 , se as ações de EPS contemplarem as necessidades da equipe, compreende-se ser preciso uma análise mais detalhada dessas iniciativas, favorecendo identificar a concepção de educação ofertada e sua capacidade de transformar o trabalho na AB.

É inegável a ênfase dada à EPS pelo PMAQ-AB. Mas a forma ou abordagem dos questionamentos também merece reflexão. Estes podem transpor os diversos limites e possibilitar uma das prerrogativas da PNEPS, a posição de 'ator' do sujeito/trabalhador de saúde, também considerando questões estruturais e locais que têm impacto no desenvolvimento da EPS e na qualidade do SUS.

\section{Relação da Atenção Básica com insti- tuições de ensino}

Do ponto de vista legal, compete à gestão do SUS o ordenamento da formação de RH para a área da saúde. Os serviços públicos de saúde, enquanto campos de prática para o ensino e a pesquisa, articulam interesses das instituições de ensino e do SUS, objetivando melhoria da qualidade do atendimento à população ${ }^{32}$.

As experiências de integração ensino-serviço no SUS foram determinantes na expansão das noções de atenção integral à saúde, de integração entre formação e trabalho, ensino e pesquisa em saúde coletiva, e de criação de projetos interprofissionais na graduação, na extensão e nas especializações em serviço ${ }^{33}$.

A PNEPS prevê, também, a organização de estruturas próprias para formação, mediante escolas de saúde pública e técnicas da rede SUS, além de centros formadores e da participação ativa das IES no seu processo de implementação. 
A integração ensino-serviço representa o trabalho pactuado e integrado por estudantes e professores dos cursos da área da saúde, com trabalhadores inseridos nos serviços de saúde e gestores, visando à qualidade da atenção, individual e coletiva, da formação profissional e ao desenvolvimento dos trabalhadores $^{34}$. Logo, há uma relação indissociável entre a rede assistencial e as instituições de ensino, que, representando um dos pilares da educação na área da saúde, acumulam a responsabilidade de formar profissionais e promover mudanças na orientação dos cursos através da implementação das diretrizes curriculares nacionais nas graduações da saúde ${ }^{28}$.

Dessa forma, cumpre abordar como o PMAQ-AB propõe a análise das equipes de $\mathrm{AB}$ e dessas instituições de ensino, e como vem ocorrendo a integração ensino e serviço.

Analisando as proposições do instrumento de avaliação, o Programa investiga o acolhimento de estudantes, professores e/ou pesquisadores em atividades de ensino, pesquisa e/ou extensão; a frequência com que ocorre; e se as atividades desenvolvidas pelos atores citados estão articuladas com o processo de trabalho da(s) equipe(s) da unidade.

O instrumento também avalia as possiblidades de ações sobre a participação da equipe em ações de EPS, quais sejam: atividades de tutoria/preceptoria e a atuação, na UBS, de alunos de graduação, especialização, residentes, entre outros. Esses questionamentos procuram pautar e reconhecer a UBS como local de formação para o SUS e de aproximação do ensino-serviço. O direcionamento do Programa é construído a partir da quantificação da frequência de comparecimento da instituição formadora, representada por estudantes, professores e/ ou pesquisadores, ao serviço de saúde e da possível articulação entre as suas demandas e o processo de trabalho da equipe.

Observa-se que a questão apresentada revela congruência com as pautas da área GES, embora restrita, atribuindo só ao trabalhador o processo de formação e qualificação.

Cabe aqui admitir o limite do instrumento em apontar características regionais quanto à estruturação da Comissão Permanente de Integração Ensino-Serviço (Cies), aspecto importante da PNEPS, tendo em vista ser o trabalhador da $\mathrm{AB}$ o sujeito entrevistado. Para tal análise, seria necessária uma investigação voltada aos atores integrantes da Cies.

Do ponto de vista das proposições efetivamente realizadas pelo PMAQ-AB, pareceria mais adequado se o instrumento estivesse organizado de modo a ir além da quantificação, ou seja, os dados poderiam indicar quem são os atores que a unidade recebe, separando-os entre estudantes, professores e/ou pesquisadores em atividades de ensino. Outro aspecto relevante seria indicar o nível de formação daquele ator relacionado, sinalizando maior ou menor aproximação com as escolas técnicas ou instituições de formação em nível superior, além dos cursos de residências.

Dessa maneira, o instrumento poderia indicar, com maior detalhamento, a realidade dos municípios e a possível relação com as instituições formadoras presentes em seu território.

No contexto da $A B$, a necessidade de mudança de paradigma na formação e qualificação profissional sobressai imperativamente, pois, nesse nível do sistema de saúde, o território é o espaço das relações entre trabalhadores e usuário. Soma-se a isso o fato de as diretrizes da $A B$ serem pautadas na construção de relações entre esses atores e no reconhecimento das realidades e dos contextos, para além da saúde, que interferem no processo saúde-doença.

A análise dessa afirmação reforça a importância do desenvolvimento de estratégias e ações que promovam a integração ensino-serviço, com a finalidade de contribuir para o processo de formação profissional na área da saúde, em consonância com as diretrizes e os princípios do SUS.

\section{Considerações finais}

$O$ trabalho na $A B$ se insere em contexto 
diverso e dinâmico, marcado por características objetivas e subjetivas. Ampliar o entendimento das questões relacionadas ao trabalho e à educação na saúde, que retratem os trabalhadores de saúde, atores-chave nesse nível de atenção, em muito pode fortalecer a estratégia.

A análise das subdimensões propostas pelo PMAQ-AB, com as diretrizes e os princípios do campo GTES, revelou aproximações, mas, também, concepções limitadas ou ausência de inclusão de importantes categorias presentes na política do trabalho e da educação na saúde.

Identificaram-se convergências nas categorias: precarização do trabalho e valorização do trabalhador, no PCCS e formas de remuneração variável. As categorias não abordadas no instrumento foram: Negociação do trabalho, Saúde do Trabalhador.

Quanto à Educação, verificou-se que o PMAQ-AB apresenta uma concepção estreita de EPS no que se refere à incorporação do conceito de EPS pelo trabalhador e nos aspectos metodológicos dos tipos de ações, descritas no instrumento. Apesar de tais limites, reconhece-se potencial de análise do instrumento quando: 1) busca dimensionar as ações de EPS e se elas contemplam as necessidades das equipes; 2) quando o instrumento apresenta a integração ensino-serviço como um padrão de qualidade para $\mathrm{AB}$; 3) quando questiona se nas unidades que recebem estudantes/professores/pesquisadores as ações desenvolvidas estão articuladas com o processo de trabalho da(s) equipe(s).
Considerando-se que mudanças no modelo assistencial requerem a valorização do trabalho e do trabalhador de saúde e que as ações relacionadas ao trabalho e à formação na saúde necessitam da articulação entre gestores, trabalhadores, instituições formadoras e controle social, torna-se fundamental investir em estratégias que busquem a melhoria, mas, também, que possibilitem o monitoramento e a avaliação dessa área. $\mathrm{O}$ PMAQ-AB converge nessa direção, apesar dos limites apontados, passíveis de superação através de um diálogo com as diretrizes, os princípios e ações de implementação propostas pela política de GTES. Nessa perspectiva, devem-se remodelar suas dimensões, superando a ausência das categorias aqui identificadas.

Afirma-se a potência de geração de informações do PMAQ-AB. O acesso à base de domínio público permite outras investigações, como uma análise descritiva dos resultados das entrevistas e como estes apontam convergências e/ou divergências com as diretrizes da política de GTES. Pela necessidade de compreensão de como tais questões estão colocadas na AB, estudos nessa direção são oportunos.

\section{Colaboradores}

Bezerra MM e Medeiros KR participaram igualmente de todas as etapas de construção do artigo. 


\section{Referências}

1. Pierantoni CR, França T, García AC. Gestão do trabalho e da educação em saúde. Rio de Janeiro: ObservaRH - IMS/UERJ; 2012.

2. Brasil. Ministério da Saúde. Portaria $n^{\circ} 2.488$, de 21 de outubro de 2011. Aprova a Política Nacional de Atenção Básica, estabelecendo a revisão de diretrizes e normas para a organização da Atenção Básica, para a Estratégia Saúde da Família (ESF) e o Programa de Agentes Comunitários de Saúde (PACS). Diário Oficial da União. 21 Out 2011.

3. Brasil. Ministério da Saúde. Programa Nacional de Melhoria do Acesso e da Qualidade da Atenção Básica-PMAQ/Manual Instrutivo [internet]. Brasília, DF: Ministério da Saúde; 2012.

4. Brasil. Ministério da Saúde. Secretaria de Atenção à Saúde. Departamento de Atenção Básica. Manual Instrutivo para as Equipes de Atenção Básica (Saúde da Família, Saúde Bucal e Equipes Parametrizadas) E NASF. Brasília, DF: Ministério da Saúde, 2013. (Série A. Normas e Manuais Técnicos).

5. Brasil. Ministério da Saúde. Portaria $n^{\circ} 645$, de 2 de outubro de 2015. Dispõe sobre o Programa Nacional de Melhoria do Acesso e da Qualidade da Atenção Básica (PMAQ-AB). Diário Oficial da União. 2 Out 2015.

6. Fausto MCR, Mendonça MHM, Giovanella L. Experiências de avaliação da Atenção Básica no Brasil: notas para um debate. In: Fausto MCR, Fonseca HR, organizadores. Rotas da atenção básica no Brasil: experiências do trabalho de campo PMAQ AB. Rio de Janeiro: Editora Saberes; 2013. p. 289-309.

7. Moraes PM, Iguti AM. Avaliação do desempenho do trabalhador como forma peculiar de prescrição do trabalho: uma análise do PMAQ-AB. Saúde debate. 2013; 37 (98): 416-426.

8. Brasil. Secretaria de Atenção à Saúde. Departamento de Atenção Básica. Nota Metodológica da Certificação das Equipes de Atenção Básica. Brasília, DF: Ministério da Saúde; 2015.
9. Pinto HA, Sousa NA, Ferla AA. O Programa Nacional de Melhoria do Acesso e da Qualidade da Atenção Básica: várias faces de uma política inovadora. Saúde debate. 2014; 38(esp):358-372.

10. Seidl M, Vieira SP, Fausto MCR, et al. Gestão do trabalho na Atenção Básica em Saúde: uma análise a partir da perspectiva das equipes participantes do PMAQ-AB. Saúde debate. 2014; 38(esp):94-108.

11. Rizzotto MLF, Gil CRR, Carvalho M, et al. Força de trabalho e gestão do trabalho em saúde: revelações da Avaliação Externa do Programa Nacional de Melhoria do Acesso e da Qualidade da Atenção Básica no Paraná. Saúde debate. 2014. 38(esp):237-251.

12. Tobar F, Yalour MR. Como fazer teses em saúde pública: conselhos e ideias para formular projetos e redigir teses e informes de pesquisa. Rio de Janeiro: Fiocruz. 2001.

13. Koster I, Machado MH. A Gestão do Trabalho e o Contexto da Flexibilização no Sistema Único de Saúde. Divulg Saúde Debate. Rio de Janeiro. 2012; 47:3344.

14. Brasil. Ministério da Saúde. Diretrizes operacionais dos Pactos pela Vida, em Defesa do SUS e de Gestão. Brasília, DF: Ministério da Saúde; 2006.

15. Brasil. Ministério da Saúde. Secretaria de Gestão do Trabalho e da Educação na Saúde. Programa de Qualificação e Estruturação da Gestão do Trabalho e da Educação no SUS - ProgeSUS. Brasília, DF: Ministério da Saúde; 2006.

16. Eberhardt LD, Carvalho M, Murofuse NT. Vínculos de trabalho no setor saúde: o cenário da precarização na macrorregião Oeste do Paraná. Saúde debate. 2015; 39(104):18-29.

17. Araújo LM, Machado MH, Vitalino HA, et al. Para subsidiar a discussão sobre a desprecarização do trabalho do SUS. Caderno de RH Saúde. 2006; 3:163-73. 
18. Costa D, Lacaz FAC, Marçal JFJ, et al. Saúde do Trabalhador no SUS: desafios para uma política pública. Rev. bras. saúde ocup. 2013; 38 (127):11-21.

19. Brasil. Ministério da Saúde. Secretaria de Gestão do Trabalho e da Educação na Saúde. Gestão do trabalho e da regulação profissional em saúde: agenda positiva do Departamento de Gestão e da Regulação do Trabalho em Saúde. Brasília, DF: Ministério da Saúde; 2009.

20. Castro JL, Vilar RLA, Liberiano FN. Gestão do Trabalho e da Educação na Saúde. Natal: UFRN; 2012.

21. Brasil. Ministério da Saúde. Portaria GM/MS nº 1.823 , de 23 de agosto de 2012. Institui, no âmbito do Sistema Único de Saúde, a Política Nacional de Atenção Integral à Saúde do Trabalhador e da Trabalhadora. Diário Oficial da União. Brasília. 24 Ago. 2012.

22. Magnago C, Pierantoni CR, França T, et al. Política de Gestão do Trabalho e Educação em Saúde: a experiência do ProgeSUS. Ciênc Saúde Colet. 207; 22 (5):1521-1530.

23. Santos EC, Sodré F, Borges LH. Mesa Nacional de Negociação Permanente: transformando o trabalho na Saúde Pública. Rev. Bras. Pesq. Saúde. 2016; 18(1):4553.

24. Martins MIC, Molinaro A. Reestruturação produtiva e seu impacto nas relações de trabalho nos serviços públicos de saúde no Brasil. Cienc Saúde Colet. 2013; 18(6):1667-1676.

25. Mendes JMR, Wunsch DS, Machado FKS, et al. Saúde do trabalhador: desafios na efetivação do direito à saúde. Argumentum. 2015; 7(2):194-207.

26. Mendes JMR, Wunsch DS. Serviço Social e a saúde do trabalhador: uma dispersa demanda. Serv. soc soc. 2011; (107):461-481.

27. Zinet, C. Condições pioram, acidentes aumentam: número de acidentes de trabalho aumenta na última década, preocupa sindicatos e organismos inter- nacionais, que culpam a forma de produção. Caros Amigos. 2012; (187):16-19.

28. Macêdo NB, Albuquerque PC, Medeiros KR. O Desafio da Implementação da Educação Permanente na Gestão da Educação na Saúde. Trab. educ. saúde. 2014; 12(2):379-40

29. Merhy EE, Franco TB. Trabalho em saúde. In: Pereira IB, Lima JCF, organizadores. Dicionário da Educação Profissional em Saúde. 2. ed. Rio de Janeiro: Fiocruz; 2009

30. França T, Medeiros KR, Belisario AS, et al. Política de Educação Permanente em Saúde no Brasil: a contribuição das Comissões Permanentes de Integração Ensino-Serviço. Ciênc Saúde Colet. 2017; 22(6):18171828.

31. Brasil. Portaria GM/ MS nº 198, de 13 de fevereiro de 2004. Institui a Política Nacional de Educação Permanente em Saúde como estratégia do SUS para a formação e o desenvolvimento de trabalhadores para o setor e dá outras providências. Diário Oficial da União. 13 Fev 2004

32. Cavalheiro MTP; Guimarães AL. Formação para o SUS e os Desafios da Integração Ensino Serviço. Caderno FNEPAS. 2011.

33. Carvalho YM, Ceccim RB. Formação e educação em saúde: aprendizados com a saúde coletiva. In: Campos GWS, Minayo MCS, Akerman M, et al., organizadores. Tratado de saúde coletiva. São Paulo. Hucitec, 2009. p. 137-170.

34. Albuquerque VS, Gomes AP, Rezende CHA. A Integração Ensino-serviço no Contexto dos Processos de Mudança na Formação Superior dos Profissionais da Saúde. Rev Bras de Educ Médica. 2008; (3):356-362.

Recebido em 29/06/2018

Aprovado em 28/08/2018

Conflito de interesses: inexistente

Suporte financeiro: Edital PPSUS - Pernambuco (CNPq/MS/SES/

Facepe - 10/2017) 